Classification

Physics Abstracts

$80.00-81.40 \mathrm{~J}-40.00-46.30 \mathrm{~J}$

\title{
Mécanismes de relaxation anélastique dans le niobium et le tantale au voisinage de l'ambiante. Influence de la teneur en oxygène et de l'écrouissage
}

\author{
D. Varchon, P. Delobelle et C. Oytana \\ Laboratoire de Mécanique Appliquée, Faculté des Sciences et des Techniques de Besançon, \\ Route de Gray, La Bouloie, 25030 Besançon Cedex, France
}

(Reçu le 7 octobre 1981, révisé le 14 décembre 1981, accepté le 22 décembre 1981)

\begin{abstract}
Résumé. - Le mécanisme de Snoek de mise en ordre par la contrainte des oxygènes interstitiels dans le niobium et le tantale (c.c.) a été étudié par la relaxation et le frottement interne. Les rôles joués par la teneur en oxygène et l'écrouissage ont plus particulièrement été approfondis.

Dans le niobium pur recuit dopé à 200 ppm, en essai de relaxation, l'amplitude de la relaxation suit une loi du type $\Delta_{\mathrm{T}}=\frac{\alpha C_{0}}{T-\beta T_{\mathrm{c}}}$ avec $\beta T_{\mathrm{c}}=220 \mathrm{~K}$ tandis que le temps de relaxation obéit à une loi d'Arrhénius identique à celle trouvée à plus haute température en frottement interne.

Lorsque la teneur en oxygène augmente, le temps de relaxation augmente avec $\Delta_{\mathrm{T}}$. Cela est dû à la réorientation de Snoek de doublets ou de triplets d'oxygène. Ainsi, pour des teneurs aussi faibles que $450 \mathrm{ppm}$, les mesures des temps de relaxation sont déjà modifiées par l'existence de doublets oxygène.

Dans le niobium commercial écroui, on remarque un effet de vieillissement thermiquement activé, relié à l'augmentation des temps de relaxation du mécanisme de Snoek-Koster. On montre que les amplitudes de relaxation de Snoek et de Snoek-Koster varient de manière à peu près proportionnelle; l'origine de ce phénomène est la migration de l'oxygène vers les dislocations. Pour les temps de vieillissement très grands, une stabilisation se produit et donne des résultats en accord avec ceux du frottement interne.

On trouve ces résultats sur le tantale.

Quelques résultats concernant le mécanisme de Snoek avec des atomes d'azote dans $\mathrm{Ta}$ et $\mathrm{Nb}$ sont également présentés.
\end{abstract}

\footnotetext{
Abstract. - The Snoek type stress induced ordering of oxygen interstitials in b.c.c. $\mathrm{Nb}$ and $\mathrm{Ta}$ has been studied through isothermic relaxation and internal friction tests. The part taken by oxygen content and by cold work is specially considered.

For annealed pure $\mathrm{Nb}$ with $200 \mathrm{ppm}$ oxygen, tested in relaxation, the relaxation strength obeys a law :

$$
\Delta_{\mathrm{T}}=\frac{\alpha C_{0}}{T-\beta T_{\mathrm{c}}}
$$

with $\beta T_{\mathrm{c}}=220 \mathrm{~K}$ while the relaxation time follows an Arrhénius law consistent with higher temperature internal friction measurements.

When oxygen content increases, the relaxation times depend on $\Delta_{\mathrm{T}}$, due to the Snoek orientation of pairs or triplets of oxygen atoms. In such a way for oxygen contents as low as $450 \mathrm{ppm}$ the relaxation time measurements is already modified by the oxygen pairs existence.

In cold worked technical niobium, thermically activated ageing is observed related to the increase of the relaxation time of the Snoek-Koster mechanism. It is shown that both relaxation strengths of the Snoek and of the SnoekKoster mechanisms are decreasing in a fairly proportional way, these phenomena are due to the oxygen migration towards the dislocations. For very long ageing times a stabilization occurs which gives results consistent internal friction ones.

These results are found again in the case of Ta.

Some results about the Snoek mechanism with nitrogen atoms in $\mathrm{Ta}$ and $\mathrm{Nb}$ are also reported.
} 
1. Introduction. - La déformation anélastique des matériaux et plus particulièrement celle associée à la remise en ordre du type Snoek des interstitiels lourds dans les métaux cubiques centrés du groupe $\mathrm{V}(\mathrm{Nb}$, $\mathrm{Ta}, \mathrm{Fe}, \mathrm{V}$...) est principalement étudiée par la technique du frottement interne [1-6]. Les études expérimentales par le traînage anélastique [7-9] et la relaxation de contrainte $[10,11]$ sont beaucoup moins nombreuses, malgré les différents avantages qu'elles présentent, à savoir : elles sont isothermes et les temps de relaxation mesurables sont grands, donc, les essais peuvent s'effectuer à plus faible température. Ainsi, les éventuels phénomènes de vieillissement thermiquement activés sont plus facilement décelables et analysables. Notons que l'approche originale de Woirgard [12] qui consiste à faire varier continuement la fréquence lors d'un essai de frottement interne isotherme, présente les mêmes avantages. Cependant, l'une des difficultés majeures de l'essai de relaxation réside dans son analyse lorsqu'il faut en déduire le spectre des temps de relaxation. On peut la résoudre soit à l'aide des méthodes numériques d'optimisation puisque l'on connaît la forme analytique des phénomènes [7-9], soit à l'aide d'une méthode fondée sur les différentes approximations de Schwartzl et Staverman [13] qui présentent l'avantage de converger rapidement et de visualiser les phénomènes. Nous rappelerons succinctement les grandes lignes de la seconde analyse publiée par ailleurs $[11,14]$ dans le paragraphe réservé aux méthodes expérimentales.

Dans le présent article, nous nous proposons donc d'étudier à l'aide de l'essai de relaxation, les différents phénomènes anélastiques associés à la présence des défauts ponctuels du type interstitiel dans le niobium et le tantale (oxygène et azote). On s'efforcera de mettre en évidence l'influence du dopage en oxygène et de l'écrouissage sur les caractéristiques du spectre de relaxation : amplitude relaxée et temps de relaxation, afin de relier les résultats obtenus aux nombreux travaux liés à la migration des interstitiels vers les dislocations.

2. Méthodes expérimentales. - 2.1 ApPareillaGE ET CARACTÉRISATION DES ÉCHANTILLONS. - Les essais de relaxation ont été réalisés sur une machine de torsion électrodynamique de très grande précision. $\mathrm{La}$ stabilité relative du couple est de $\pm 3 \times 10^{-5}$ pendant $10^{5} \mathrm{~s}$ pour une déformation superficielle de l'ordre de $10^{-5}$ [15]. Les échantillons sont des fils de 0,5 à $1 \mathrm{~mm}$ de diamètre et de $600 \mathrm{~mm}$ de longueur, la température pouvant varier de $-20^{\circ}$ à $+200^{\circ} \mathrm{C}$.

Parallèlement à ces essais, quelques expériences de frottement interne au voisinage de $0,3 \mathrm{~Hz}$ ont été réalisées sur un pendule de torsion inversé asservi permettant d'obtenir directement le spectre de frottement interne $Q^{-1}$ en fonction de la température.

Les caractéristiques des fils et des traitements de recuit de dopage sont données ci-dessous (tableau I). Il faut noter que les dosages ont été réalisés par spectroscopie de masse (S.M.), ou par frottement interne (F.I.), en utilisant les relations de Ahmad et Szkopiak pour le niobium [5] ou de Szkopiak et Eliasz pour le tantale [4].

2.2 ANAlyse Des Courbes DE RelaXation. Pour un solide linéaire standard (type Zener) et dans le cas d'un spectre discret mais fini de temps de relaxation $\tau_{i}$, la variation de la contrainte dans le temps s'écrit :

$$
\sigma(t)=\sigma_{\mathrm{f}}+\sum_{i} \Delta \sigma_{i} \exp -\frac{t}{\tau_{i}} .
$$

Pour un seul temps de relaxation, (1) se réduit à l'expression

$$
\sigma(t)=\sigma_{\mathrm{f}}+\Delta \sigma \exp -\frac{t}{\tau}
$$

dans laquelle $\sigma_{\mathrm{f}}$ et $\sigma_{0}=\Delta \sigma+\sigma_{\mathrm{f}}$ sont respectivement les contraintes pour les temps $t=\infty$ et $t=0$.

Pour un spectre continu, l'équation (1) peut se mettre sous la forme,

$$
\sigma(t)=\sigma_{\mathrm{f}}+\Delta \sigma \int_{-\infty}^{+\infty} H(\operatorname{Ln} \tau) \exp -\frac{t}{\tau} \mathrm{d} \operatorname{Ln}(\tau)
$$

qui permet, à l'aide du calcul symbolique, de remonter aux approximations successives de $H(\operatorname{Ln} \tau)$ de Schwartzl et Staverman [13] :

$$
H_{p}(\operatorname{Ln} \tau)=\frac{(-1)^{p}}{\Delta \sigma(p-1) !} p^{p} \tau^{p-1}\left[\frac{\mathrm{d}^{p} \sigma(t)}{\mathrm{d}(\operatorname{Ln} t)^{p}}\right]_{t=\tau p}
$$

de précision croissante avec $p$. Elles présentent l'avantage de faire intervenir $\sigma(t)$ par l'intermédiaire de ses dérivées d'ordre $p$ par rapport à $\operatorname{Ln} t$ et n'exigent donc pas la connaissance de toute la courbe $\sigma(t)$ et en particulier de $\sigma_{(\infty)}$. On montre alors [11, 14], en comparant un spectre théorique du type (1), avec deux temps de relaxation par exemple, aux approximations successives $H_{1}, H_{2}$ et $H_{3}$ données par (4) pour $p=1,2,3$ que les maxima de $H_{p}(\operatorname{Ln} \tau)$ coïncident dès la première approximation avec les temps de relaxation $\tau_{i}$. La précision liée à cette convergence rapide est donc bonne et dans la suite de l'étude, la première approximation $H_{1}$ sera souvent suffisante. De plus, on peut montrer qu'il est possible de séparer deux maxima tant que $\tau_{2} / \tau_{1} \geqq 4$ pour $\Delta \sigma_{1}=\Delta \sigma_{2}$, et que ce pouvoir de résolution, comparable à celui $\mathrm{du}$ frottement interne, augmente ensuite rapidement avec $p$ (séparer signifiant avoir deux maxima distincts sans décomposition). Nous utiliserons cette propriété dans la suite de l'étude.

En pratique, le processus opératoire peut se résumer de la manière suivante :

- détermination expérimentale de $\sigma=f(t)$;

- calcul et tracé de

$$
Y(t)=\frac{\sigma_{0}-\sigma(t)}{\sigma_{0}}
$$


Tableau I $a$ - Nb pur;99,996 \%, $\varnothing 0,54 \mathrm{~mm}$.

\begin{tabular}{|c|c|c|c|c|c|}
\hline $\begin{array}{l}\text { Température } \\
\text { de recuit }\end{array}$ & $\begin{array}{l}\text { Pression } \\
\text { (torr) }\end{array}$ & Temps & $\begin{array}{l}\text { ppm oxygène } \\
\text { en poids }\end{array}$ & Méthode & No \\
\hline - & - & - & - & - & - \\
\hline Non recuit & - & - & 35 & F.I. & 0 \\
\hline $1150^{\circ} \mathrm{C}$ & $10^{-5}$ & $3 \mathrm{~h}$ & 200 & S.M. & 1 \\
\hline - & $10^{-5}$ & $5 \mathrm{~h}$ & 450 & S.M. & 2 \\
\hline - & $10^{-4}$ & $9 \mathrm{~h}$ & 990 & S.M. & 3 \\
\hline - & $10^{-3}$ & $5 \mathrm{~h}$ & 3100 & S.M. & 4 \\
\hline - & $10^{-2}$ & $2 \mathrm{~h}$ & 3960 & S.M. & 5 \\
\hline - & $5 \times 10^{-3}$ & $5 \mathrm{~h}$ & 5400 & F.I. & 6 \\
\hline - & $10^{-2}$ & $4 \mathrm{~h}$ & 7260 & S.M. & 7 \\
\hline
\end{tabular}

Ib. - $\mathrm{Nb}$ commercial, $\varnothing 0,8 \mathrm{~mm}$, écroui à $45 \%$ en filière

Ic. - Ta pur, $99,996 \%, \varnothing 0,54 ;$ non recuit, $\simeq 50 \mathrm{ppm}$ d'oxygène, I.F. - recuit $5 \mathrm{~h}$ sous $10^{-5}$ torr, $\simeq 130 \mathrm{ppm}$ d'oxygène, I.F.

$\begin{array}{lrlrlll}\mathrm{H}_{2} \leqq 75 & \mathrm{Ta} & 1 & 270 & \text { S.M. } & \mathrm{O} \simeq 90 & \text { F.I. } \\ \mathrm{N} \leqq 200 & \mathrm{~W} & 646 & & \mathrm{~N} \simeq 100 & \text { F.I. } \\ \mathrm{O}_{2} \leqq 125 & \mathrm{~Pb} & 19 & & & \\ \mathrm{Si} & 293 & \mathrm{Zn} & 12 & & & \\ \mathrm{~S} & 245 & \mathrm{Fe} & 131 & & & \\ \mathrm{Ti} & 212 & \mathrm{Li} & 81 & & & \\ \mathrm{Mo} & 130 & \mathrm{~F} & 61 & & & \end{array}$

Id. - Tantale commercial, $\varnothing 0,6 \mathrm{~mm}$, traitement de recuit

$\begin{array}{lrlll}1 & 30^{\prime} \text { à } 1050^{\circ} \mathrm{C} \text { sous } 3 \times 10^{-5} \text { plus } 40^{\prime} & 1050^{\circ} \mathrm{C} & 10^{-3} \text { torr } & \\ 2 & -45^{\prime} & 1050- & 10^{-2}- & \text { oxygène } \\ 3 & -1 \mathrm{~h} 30 & 1050- & 10^{-2}- & \text { non dosée } \\ 4 & -1 \mathrm{~h} 30 & 1050- & 10^{-2}- & \end{array}$

Ie. - Ta commercial, $\varnothing 0,6 \mathrm{~mm}$, recuit durant $45 \mathrm{~min}$. à $800{ }^{\circ} \mathrm{C}$ sous $2 \times 10^{-5}$ torr puis écroui à $40 \%$

$\begin{array}{rrr}\mathrm{O} & 105 & \text { I.F. } \\ \mathrm{N} & \simeq 20 & \text { I.F. }\end{array}$

Eléments métalliques non dosés

à partir d'une trentaine de points équidistants en échelle logarithmique;

- lissage de $Y$ par un polynôme $p_{n}(t)$ de degré inférieur ou égal à dix.

- utilisation des formules (4), qui appliquées à $Y$ donnent $H_{p}(\operatorname{Ln} \tau) / \sigma_{0}$

- tracé des approximations $H_{1}$ et $H_{2}$.

L'ensemble du traitement numérique est réalisé sur calculatrice H.P. 9830 couplée à une table traçante.

3. Résultats expérimentaux et analyse. - 3.1 RelaXation dans Le NIOBIUM. - i) Cas du niobium pur recuit. - Identification du phénomène et évolution du temps de relaxation avec la température (traitement de recuit $\mathrm{n}^{\circ} 1$, tableau $\mathrm{I} a$ ).

La figure 1 donne un exemple de la représentation et de l'évolution avec la température de $Y$ et de $H_{1}(\operatorname{Ln} \tau)$ en fonction de $\operatorname{Ln} t$. On constate que $H_{1}$ présente un maximum bien marqué dont l'amplitude et la position décroissent avec la température.

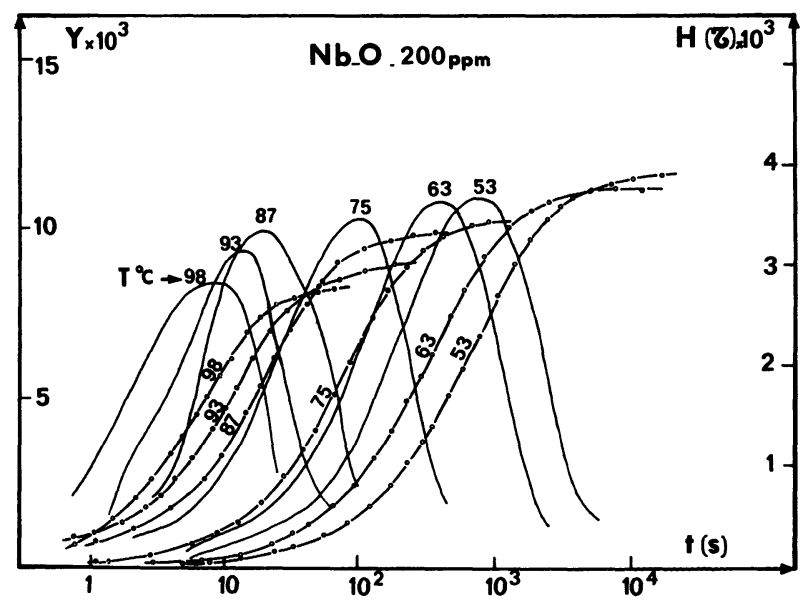

Fig. 1. - Niobium pur. Courbes de $Y$ et de $H_{1}(\tau)$ pour quelques isothermes.

[Pure niobium. Curves of $Y$ and $H_{1}(\tau)$ for various temperatures.] 
L'analyse de la position des maxima à l'aide du diagramme d'Arrhénius Ln $\sigma=f(1 / T)$ (Fig. 2, droite (1) permet d'identifier le phénomène et de montrer que $\tau$ correspond au temps de relaxation de la remise en ordre du type Snoek de l'oxygène dans le niobium [13]. En effet, dans cette représentation, nos points expérimentaux se trouvent sensiblement dans le prolongement des points déterminés en frottement interne à plus haute température correspondant à la remise en ordre de Snoek [13]. On reporte également les points obtenus en frottement interne. Finalement, on aboutit à

$$
\tau=\tau_{0} \exp \frac{W_{\mathrm{S}}}{k T}
$$

avec

$\tau_{0} \simeq 1,9 \times 10^{-14} \mathrm{~s}$ et $W_{\mathrm{s}}=1,045 \times 10^{5} \mathrm{~J} \cdot \mathrm{mole}^{-1}$, valeurs comparables à celles obtenues par Boratto et Reed-Hill [9] en traînage anélastique à savoir; $\tau_{0}=5,4 \times 10^{-15} \mathrm{~s}$ et $W_{\mathrm{s}}=1,096 \times 10^{5} \mathrm{~J} \cdot \mathrm{mole}^{-1}$.

Nous tenterons d'expliciter la légère différence à la lumière des résultats présentés ultérieurement.

Il faut remarquer qu'il n'existe que très peu de mesures directes de temps de relaxation à si basse température : $23^{\circ} \leqq T \leqq 120^{\circ} \mathrm{C}$.

ii) Etude de l'amplitude relaxée en fonction de la température et de la teneur en oxygène. - Compte tenu de la technique même du frottement interne, les résultats expérimentaux concernant l'évolution de l'amplitude totale relaxée $\Delta_{\mathrm{T}}$ avec la température

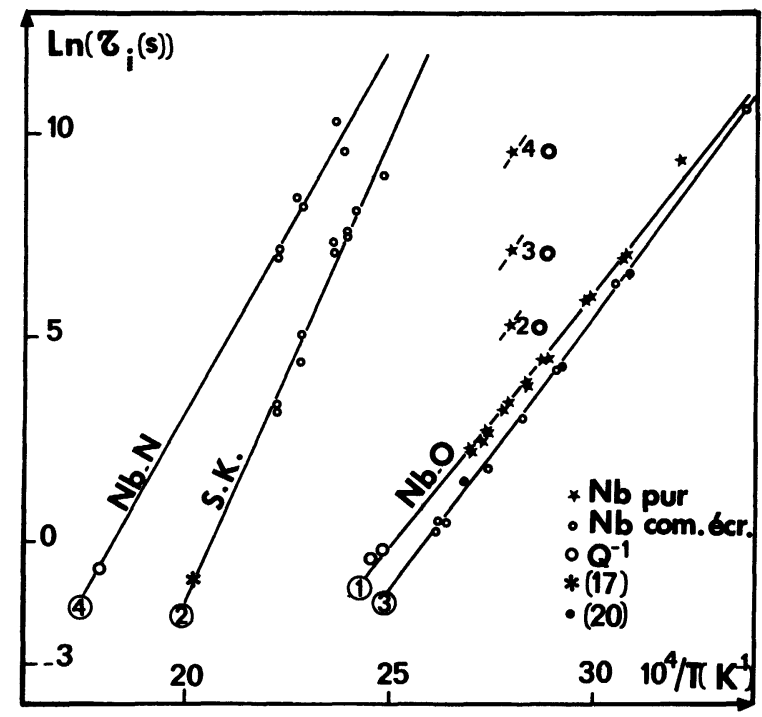

Fig. 2. - Diagramme d'Arrhénius correspondant aux temps de relaxation $\tau_{i}$ des différents phénomènes observés dans le niobium (pic de Snoek et pic de Snoek-Koster).

[Arrhénius diagram for the $\tau_{i}$ relaxation times of the various observed phenomena in $\mathrm{Nb}$-Snoek peaks and SnoekKoster peaks).] sont peu nombreux et souvent contradictoires [13]. Cependant, d'un point de vue théorique, il se dégage qu'une loi du type Curie-Weiss,

$$
\Delta_{\mathrm{T}}=\frac{\alpha C_{0}}{T-\beta T_{\mathrm{c}}}
$$

devrait s'appliquer [13]. $\beta T_{\mathrm{c}}$ est la température critique pour laquelle l'ordre se rétablit spontanément et dépend d'un coefficient d'interaction entre atomes d'oxygène et de la concentration $C_{0}$ d'oxygène libre. Compte tenu de l'incertitude des mesures de $\Delta_{\mathrm{T}}$ et de la faible plage de température couverte par les expériences, il est très difficile de déterminer $\beta T_{\mathrm{c}}$ qui est souvent négligé. Cependant, Gibala et Wert [7] ont trouvé $T_{\mathrm{c}} \simeq 82 \mathrm{~K}$ pour Nb-O tandis que Powers et Doyle [10] donnent $T_{\mathrm{c}} \simeq 105 \mathrm{~K}$ pour Ta-O. Sur la figure $3 a$, on reporte

$$
\Delta_{\mathrm{T}}=\frac{Y_{(\infty)}}{1-Y_{(\infty)}}=\frac{H_{1} e}{1-Y_{\infty}}
$$

$\left(\operatorname{avec} Y_{(\infty)}=Y_{(t)}\right.$ pour $\left.t \rightarrow \infty\right)$ en fonction de $1 / T$ et l'on vérifie cette difficulté expérimentale, $\beta T_{\mathrm{c}}$ est trop faible pour que la faible plage de température $(320 \mathrm{~K}-400 \mathrm{~K})$ permette de le mesurer, conduisant alors à :

$$
\Delta_{\mathrm{T}}=\frac{\alpha^{\prime} C_{0}}{T} .
$$

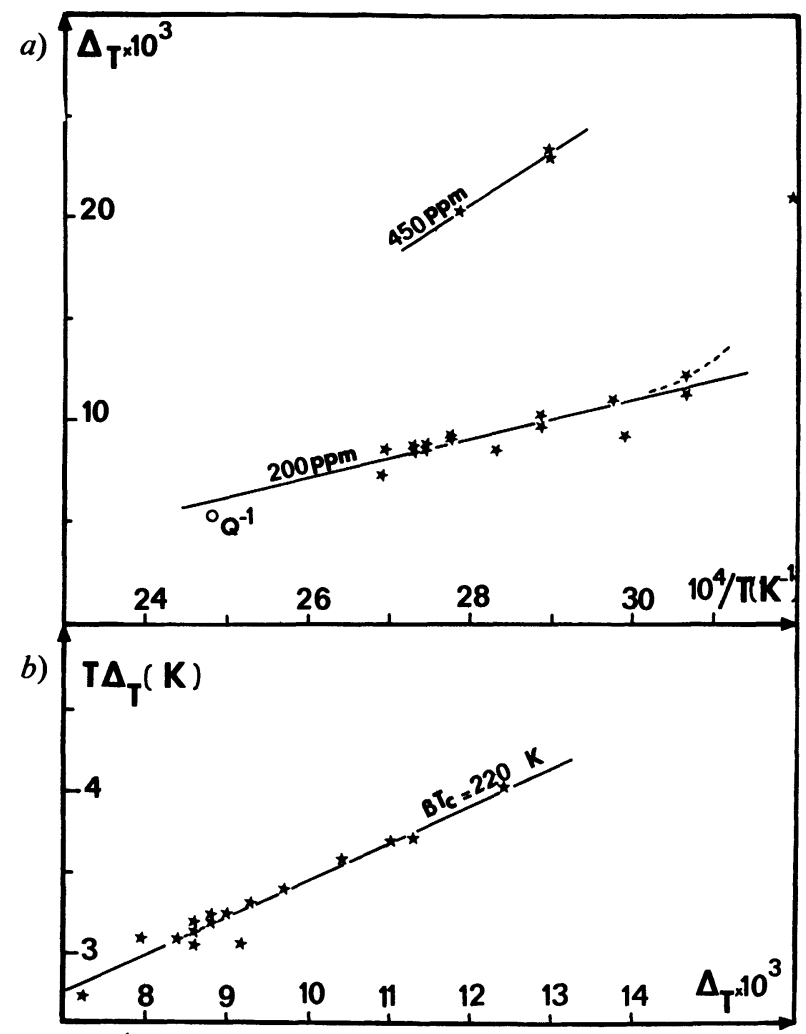

Fig. 3. - $-a$ ) Variation de l'amplitude du mécanisme de Snoek $\Delta_{\mathrm{T}}$ avec la température. b) Détermination de $\beta T_{\mathrm{c}}$.

(a) Relaxation strength of the (Snoek mechanism versus temperature. $b$ ) Determination of $\beta T_{c}$.] 
Il est cependant possible d'approximer $\beta T_{\mathrm{c}}$ en traçant $T \Delta_{\mathrm{T}}=f\left(\Delta_{\mathrm{T}}\right)$ à $C_{0}=$ Cte, ce qui est réalisé sur la figure $3 b$ où l'on trouve $\beta T_{\mathrm{c}} \simeq 220 \mathrm{~K}$ pour $220 \mathrm{ppm}$. Quelques essais ont été réalisés sur des échantillons dopés à $450 \mathrm{ppm}$ et conduisent à $\beta T_{\mathrm{c}} \simeq 270 \mathrm{~K}$, ce qui montre qu'effectivement ce terme dépend fortement de la concentration et que $T_{\mathrm{c}} \simeq 100 \mathrm{~K}$ pour des échantillons très purs. Comme nous le verrons ci-dessous, la valeur élevée de $270 \mathrm{~K}$ ne semble pas anormale puisque dès $300 \mathrm{ppm}$ l'interaction entre atome d'oxygène est très forte vers l'ambiante. Dans le tableau ci-dessous (Tableau II),

Tableau II

$\begin{array}{cc}\Delta_{\mathrm{T}} / \text { at. } \% & T \mathrm{~K} \\ \overline{0,09} & -\overline{418} \\ 0,078 \leqq \Delta \leqq 0,088 & 400 \leqq T \leqq 450 \\ 0,26 & 431 \\ 0,075 \leqq \Delta \leqq 0,11 & 327 \leqq T \leqq 370 \\ 0,09 \leqq \Delta \leqq 0,11 & 344 \leqq T \leqq 360\end{array}$

La figure 4 montre, pour une même isotherme, l'évolution de $Y$ et de $H_{1}$ avec la teneur en oxygène $C_{0}$ obtenue par différents recuits de dopage (\$2) : $Y$ et $H_{1}$ croissent fortement avec $C_{0}$ alors que $\tau_{\mathrm{r}}$ se déplace vers les temps longs. Les figures $5 a$ et $5 b$ représentent respectivement l'évolution

$$
\operatorname{Ln} \Delta_{\mathrm{T}}=f\left(\operatorname{Ln} C_{0}\right) \quad \text { et } \quad \operatorname{Ln} \Delta_{\mathrm{T}}=f\left(\operatorname{Ln} \tau_{\mathrm{r}}\right),
$$

ainsi que le point obtenu par Woirgard par frottement interne isotherme [12]. On vérifie ainsi que

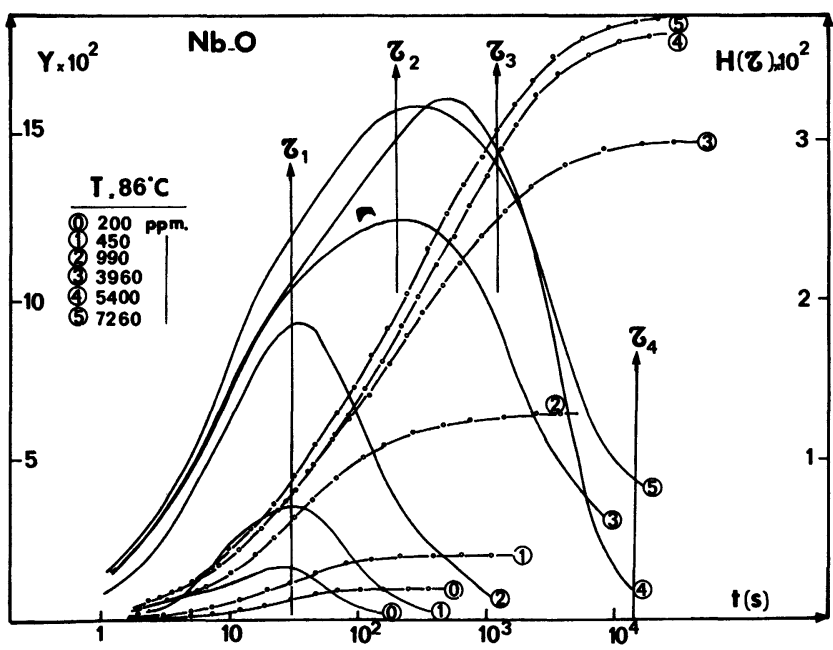

Fig. 4. - Evolution de $Y$ et de $H_{1}(\tau)$ en fonction du pourcentage en oxygène.

[Evolution of $Y$ and $H_{1}(\tau)$ as functions of the oxygen content.] nous confrontons nos résultats à ceux disponibles dans la littérature et obtenus en frottement interne. Si l'on fait abstraction du terme $\beta T_{c}$, on montre ainsi qu'il y a bon accord, du moins avec deux auteurs, et que l'amplitude relaxée par pourcent atomique est de l'ordre de 0,04 (2) vers $418 \mathrm{~K}$. A l'aide de l'équation complète (7), on trouve une valeur assez proche $\Delta_{\mathrm{T}} /$ at. $\% \simeq 0,05(5)$ pour $418 \mathrm{~K}$ car on a sensiblement $\alpha^{\prime} \simeq 1,7 \alpha$. La première colonne du tableau donne le $\Delta_{\mathrm{T}}$ mesuré par atome, la $3^{\mathrm{e}}$ colonne celui qui en est déduit par la relation (9) (ou (7) quand $\beta T_{c}$ est indiqué).

$$
\begin{array}{cc}
\Delta_{\mathrm{T}} / \text { at. } \% \text { à } 418 \mathrm{~K} & \text { Réfs. } \\
\frac{-}{0,09} & - \\
0,08 & {[13]} \\
\simeq 0,26 & {[12]} \\
\simeq 0,04(2) & {[5]} \\
0,05(5) \text { pour } \beta T_{\mathrm{c}} & \text { Présente étude } \\
=200 \mathrm{~K} & 200 \mathrm{ppm} \\
\simeq 0,04(7) & \\
& 450 \mathrm{ppm}
\end{array}
$$

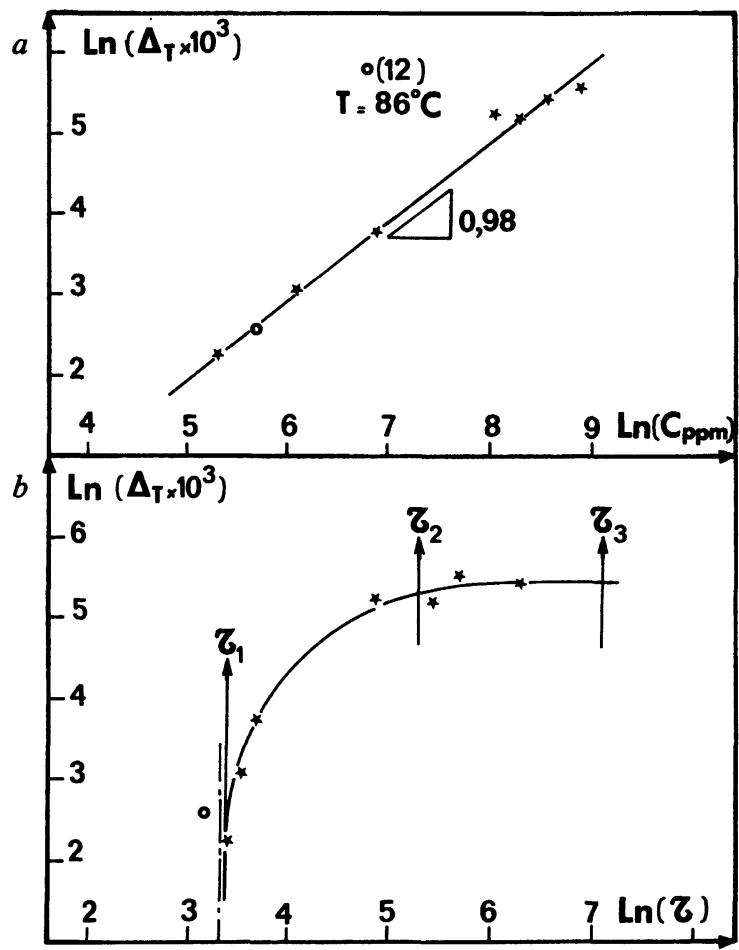

Fig. 5. - a) Vérification de la proportionnalité entre l'amplitude $\Delta_{\mathrm{T}}$ et la concentration $C$. b) Relation existant entre $\Delta_{\mathrm{T}}$ et $\tau_{\mathrm{r}}$ lorsque la concentration $C$ augmente. Positionnement des temps $\tau_{1}, \tau_{2}$ et $\tau_{3}$ des configurations 0,20 et 30 .

[a) A verification of the proportionality between the relaxation strength $\Delta_{\mathrm{T}}$ and the oxygen content $C . b$ ) Relation between $\Delta_{\mathrm{T}}$ and $\tau_{\mathrm{r}}$ when the oxygen content $C$ increases. Positions of time $\tau_{1}, \tau_{2}$ and $\tau_{3}$ corresponding to the configurations 0.20 and 30 .] 
l'amplitude totale relaxée $\Delta_{\mathrm{T}}$ est bien proportionnelle à $C_{0}$ conformément à l'équation (7) (résultat classique), alors que le temps de relaxation croît avec $\Delta_{\mathrm{T}}$ donc avec $C_{0}$. Ce dernier phénomène est dû à la remise en ordre du type Snoek des atomes d'oxygène associés en paires, triplets voir même quadruplets lorsque la teneur globale en oxygène croît $[5,7,10]$. Ainsi, la position du maximum du spectre $\tau_{\mathrm{r}}$ n'a plus de signification puisque la relaxation est imputable à plusieurs mécanismes dont les temps de relaxation sont différents (éq. (1)). Même pour les plus faibles teneurs $(\simeq 450 \mathrm{ppm}), \tau_{\mathrm{r}}$ est très légèrement fonction de $C_{0}$ ce qui montre que les atomes d'oxygène s'associent par paire. Cette constatation est cohérente avec la valeur de $\beta T_{\mathrm{c}} \simeq 200 \mathrm{~K}$ obtenue précédemment et montre que pour mesurer uniquement le temps de relaxation des atomes non appariés, il faut des échantillons de bonne pureté ( $\left.C_{0} \leqq 100 \mathrm{ppm}\right)$, ce qui est souvent incompatible avec les traitements de recuit ou d'adoucissement sous vide secondaire $\left(\simeq 10^{-6}\right.$ torr).

Si l'on considère que les mécanismes de remise en ordre des différentes configurations opèrent de manière indépendante, il est possible de décomposer le spectre global $H_{1}$ en une somme de $n$ spectres, à l'aide des formules (1) et (4) $(n \leqq 4)$. Les résultats de ces décompositions, temps de relaxation $\tau_{i}$ et amplitude relaxée $\Delta_{i}$ de chaque configuration, sont donnés dans le tableau III.

Tableau III. - Décomposition pour $T=86^{\circ} \mathrm{C}$.

\begin{tabular}{|c|c|c|c|c|c|c|c|c|}
\hline ppm & $\Delta_{\mathrm{T}} \times 10^{3}$ & $\tau_{1}(\mathrm{~s})$ & $\tau_{2}$ & $\tau_{3}$ & $\tau_{4}$ & $\Delta_{1} \times 10^{3}$ & $\Delta_{2}$ & $\Delta_{3}$ \\
\hline$\overline{200}$ & -9 & $\overline{29}$ & - & - & - & $\overline{9}$ & - & - \\
\hline 450 & 20 & 30 & 190 & - & - & 17 & 4 & - \\
\hline 990 & 42 & 33 & 200 & - & - & 25 & 17 & $(-)$ \\
\hline 3960 & 176 & 27 & 200 & 1200 & - & 37 & 56 & 57 \\
\hline 5400 & 219 & 30 & 200 & 1250 & $(-)$ & 37 & 52 & 97 \\
\hline 7260 & 250 & 30 & 190 & 1200 & 15000 & 44 & 56 & 87 \\
\hline
\end{tabular}

On constate ainsi que les résultats convergent de manière significative et que pour cette isotherme, les temps de relaxation des oxygènes simples, des paires et des triplets sont respectivement 30,200 et $1200 \mathrm{~s}$ (Fig. $5 b$ ), valeurs comparables à celles obtenues en trainage anélastique par Gibala et Wert [7].

Pour le plus fort dopage, il est possible de trouver un quatrième temps de relaxation mais celui-ci est peu précis et se situe vers $15000 \mathrm{~s}$ (Fig. 4). En ce qui concerne les amplitudes relaxées $\Delta_{i}$ des différentes configurations, malgré une certaine dispersion et dans les limites de l'étude, il est visible qu'elles tendent successivement à se saturer, ce phénomène ayant déjà été observé par Ahmad et Szkopiak en frottement interne [6]. Cette constatation est compatible avec le fait que la configuration d'ordre $n$ se développe au détriment de celle d'ordre $(n-1)$, ce qui peut s'exprimer à l'aide de la Loi d'Action de Masse qui régit les relations d'équilibre entre les différentes configurations possibles :

$$
\Delta_{\mathrm{T}}=A C_{0}=\sum_{i=1}^{n} \Delta_{i}=\sum_{i=1}^{n} a_{i} C_{1}^{i},
$$

$C_{1}$ étant la concentration en oxygène libre.

iii) Influence de l'écrouissage, cas du niobium de pureté commerciale. - Pour mettre en évidence l'influence de l'écrouissage sur la relaxation de Snoek nous avons réalisé, pour une même isotherme plusieurs relaxations successives sur un même échantillon de niobium commercial écroui à $45 \%$ (tréfilage). La figure 6 reporte, pour quelques isothermes, l'évolution

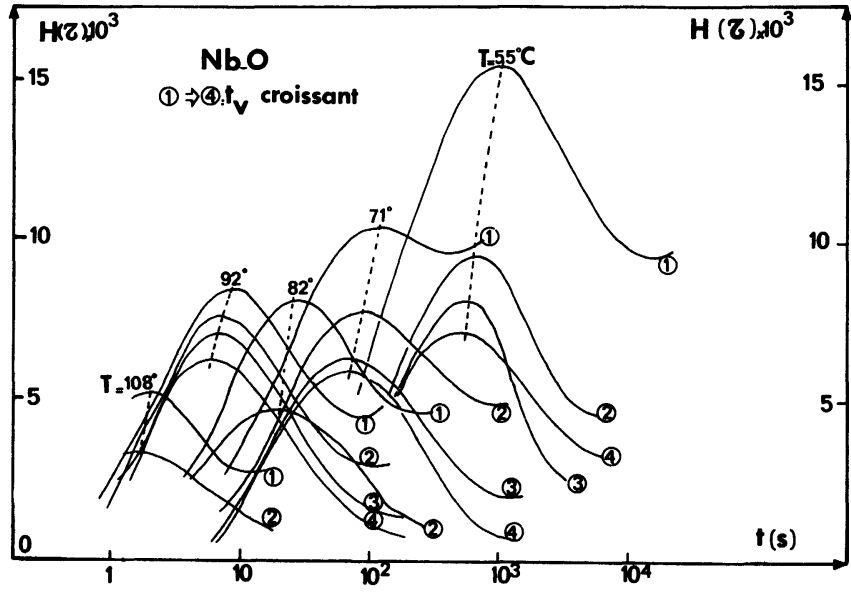

Fig. 6. - Vieillissement du mécanisme de Snoek $\left(H_{1}(\tau)\right)$ pour quelques isothermes.

[Ageing of the Snoek $\left(H_{1}(\tau)\right)$ mechanism for various isothermal experiments.]

globale de $H_{1}$ au cours des relaxations successives. On constate que $H_{1}$ décroît d'une relaxation à la suivante, alors que $\tau_{\mathrm{r}}$ décroît légèrement vers les temps courts jusqu'à ce que le phénomène semble se stabiliser et ceci d'autant plus rapidement que la température est élevée. Cette évolution est caractéristique d'un phénomène de vieillissement thermiquement activé et peut s'expliquer en partie par la présence et le déplacement du pic d'écrouissage (Pic de Snoek-Köster ou S.K.) au cours du temps [13, 16, 17]. Cet effet est bien visible sur la figure 7 où le pic S.K. se translate depuis le pic de Snoek vers les temps 


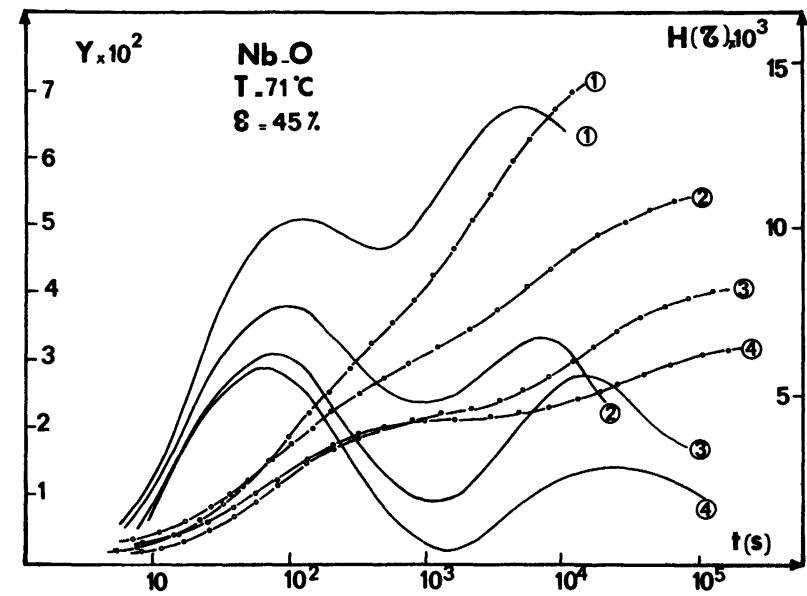

Fig. 7. - Mise en évidence de la translation du pic S.K. au cours des relaxations successives.

[Observations of the Snoek-Koster relaxation time during consecutive relaxation tests.]

longs au cours des relaxations successives. Nous n'approfondirons pas l'étude de la cinétique de migration du pic d'écrouissage, déjà publiée par ailleurs [16], nous reportons simplement, sur la représentation d'Arrhénius de la figure 2, la position stable de son temps de relaxation pour un temps de vieillissement très long, ainsi que le point obtenu par Lamotte et Wert [17] (droite (2)). On aboutit ainsi à $\tau_{0} \simeq 5 \times 10^{-22} \mathrm{~s}$ et

$$
W_{\text {S.K. }}=1,97 \times 10^{5} \mathrm{~J} . \mathrm{mole}^{-1},
$$

la valeur de l'énergie d'activation $W_{\text {s.K. }}$ étant égale à celle reportée par Boone et Wert pour le niobium [18].

$\mathrm{Si}$ on évalue l'évolution de l'amplitude totale relaxée $\Delta_{\mathrm{s}}$ du mécanisme de Snoek en fonction du temps, ce qui est facilement réalisable sans trop d'incertitude puisque

$$
Y_{\mathbf{S}}(\infty)=\left(1-\frac{1}{e}\right)^{-1} Y_{\mathbf{S}}(\tau)
$$

et que la dérive est très faible, $\left(Y_{\mathrm{S}}(\tau)=Y_{\mathrm{S}}(t)\right.$ pour $t=\tau$ ), on constate qu'elle décroît assez rapidement pour se stabiliser vers les temps longs et ceci d'autant plus rapidement que la température est élevée (Fig. 8a). Ce phénomène de vieillissement dans les matériaux cubiques centrés a été largement étudié soit par la méthode du retour de limite d'élasticité [19-24], soit par la recouvrance du frottement interne $[4,25,26]$ et est attribué à la migration à longue distance des interstitiels vers les dislocations. Ainsi, si l'on prend une cinétique du premier ordre,

$$
\frac{\mathrm{d} q}{\mathrm{~d} t}=(1-q) f(t),
$$

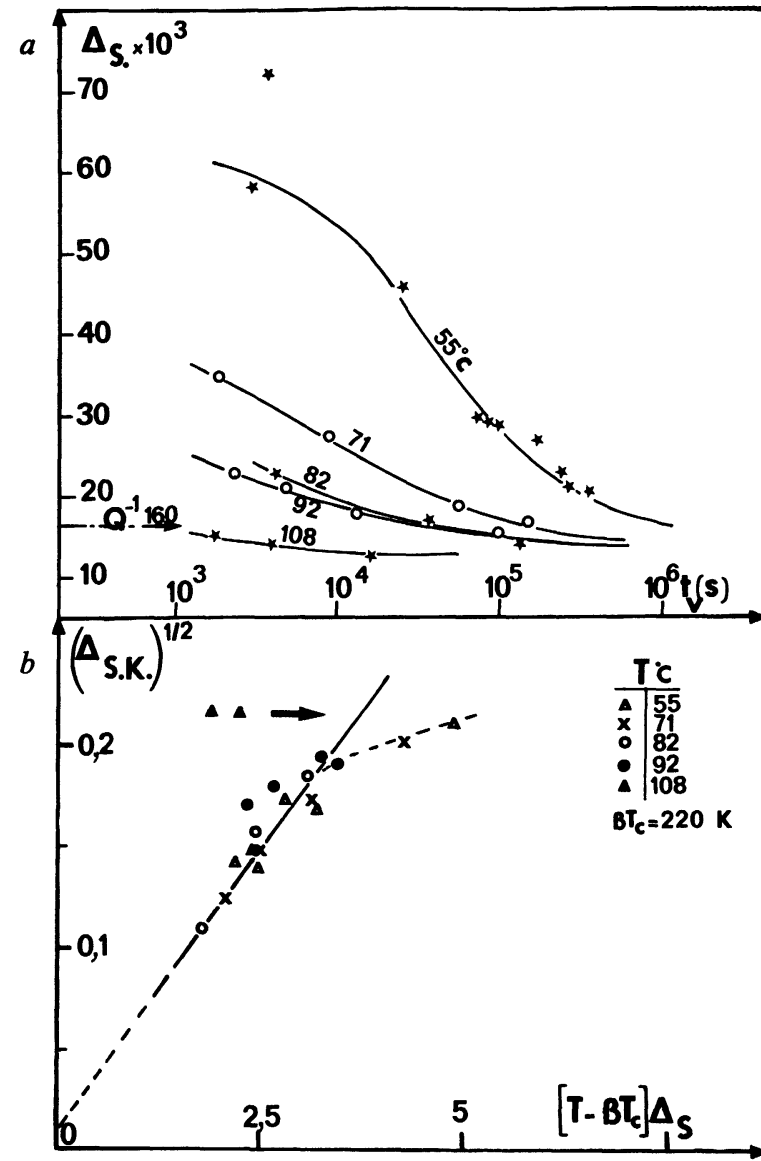

Fig. 8. $-a$ ) Décroissance de l'amplitude relaxée $\Delta_{\mathrm{S}} \mathrm{du}$ réarrangement de Snoek avec le temps de vieillissement $t_{\mathrm{v}}$. b) Relation existant entre les amplitudes des pics S.K. et de Snoek au cours du vieillissement, et pour quelques isothermes. Vérification de l'équation (19).

[a) Relaxation strength $\Delta_{\mathrm{S}}$ decrease of the Snoek mechanism with the ageing time $t_{\mathrm{v}} . b$ ) Relationship between the relaxation strengths of the Snoek-Koster and Snoek mechanisms, during ageing at various temperatures. Verification of equation (19).]

loi introduite par Harper [25]

$$
\left(q=\frac{C_{\mathrm{d}}(t)-C_{\mathrm{d}}(0)}{C_{\mathrm{d}}(\infty)-C_{\mathrm{d}}(0)}\right)
$$

$C_{\mathrm{d}}(\infty)$ et $C_{\mathrm{d}}(0)$ étant les concentrations en oxygène sur les dislocations aux temps $t=\infty$ et $t=0$ et si l'on choisit $f(t)$ telle qu'aux temps courts on ait

$$
q=\alpha\left(\frac{D t}{k T}\right)^{\frac{2}{m+2}} \text { [27] }
$$

on trouve la solution de (11),

$$
q=\left[1-\exp \left(-\alpha \frac{D t}{k T}\right)^{\frac{2}{m+2}}\right]
$$


expression qui sature pour $t \rightarrow \infty$. Dans cette relation $\alpha$ est une constante, $D$ le coefficient de diffusion de l'impureté considérée dans le métal de base et $m$ un coefficient caractéristique du potentiel d'interaction dislocations-interstitiels de la forme $V(r)=K_{m} / r^{m}$ ( $r$ est la distance entre l'interstitiel et la dislocation considérée). On peut noter que pour $m=1$, on retrouve la loi de Bilby-Cottrell en $t^{2 / 3}$ valable pour les temps courts. Puisque la concentration totale en oxygène $C_{\mathrm{T}}$ est constante, on a

$$
C_{\mathrm{T}}=C_{0}+C_{\mathrm{d}},
$$

donc la loi (13) avec les amplitudes de Snoek $\Delta_{\mathrm{S}}$ s'applique,

$$
q=\frac{\Delta_{\mathrm{S}}(t)-\Delta_{\mathrm{S}}(0)}{\Delta_{\mathrm{S}}(\infty)-\Delta_{\mathrm{S}}(0)}
$$

mais cette fois avec $\Delta_{s}(0) \geqq \Delta_{s}(\infty)$. Présentement, compte tenu d'une part du temps d'établissement et de stabilisation de la température au sein de l'installation ( $\simeq 1 \mathrm{~h}$ ) et d'autre part, du dopage assez conséquent des échantillons, nos résultats se situent déjà dans la phase de saturation de la loi (13) (Fig. 8a). On peut remarquer qu'en frottement interne, lorsque le maximum apparaît vers $160^{\circ} \mathrm{C}$, l'amplitude relaxée est proche de sa valeur minimum.

Nous avons estimé la valeur de $m$ en mettant l'équation (13) sous la forme :

$$
\begin{aligned}
\operatorname{Ln}\left[\Delta_{\mathrm{S}}(t)-\Delta_{\mathrm{S}}(\infty)\right]=\operatorname{Ln}\left[\Delta_{\mathrm{S}}(0)-\Delta_{\mathrm{S}}(\infty)\right]- & \\
& -\left(\frac{\alpha D t}{k T}\right)^{\frac{2}{m+2}},
\end{aligned}
$$

et en cherchant à linéariser les représentations

$$
\operatorname{Ln}\left[\Delta_{\mathrm{S}}(t)-\Delta_{\mathrm{S}}(\infty)\right]=f^{\left(t^{\frac{2}{m+2}}\right)}
$$

en ajustant le paramètre $m$. La précision est faible et l'on aboutit à $1,5 \leqq m \leqq 2$. Cet encadrement semble raisonnable puisque divers auteurs rapportent des valeurs comprises entre 1 et 4 [20-26].

Parallèlement à la décroissance de l'amplitude de la relaxation de Snoek, on observe une décroissance de celle de la relaxation de Snoek-Koster $\Delta_{\text {s.K. }}$. Cette décroissance est peu observable aux températures où le pic d'écrouissage de Snoek-Koster apparaît en frottement interne. Elle est quelquefois interprétée de la manière suivante (par exemple en [13]) : la théorie de Schoeck [28] dont le principe est habituellement admis pour interpréter le pic de SnoekKoster prévoit :

$$
\Delta_{\mathrm{S} . \mathrm{K} .}=\beta \Lambda l^{2}
$$

où $\beta$ est un facteur géométrique de l'ordre de 0,1 , $\Lambda$ la densité de dislocations épinglées et $l$ la distance moyenne entre deux points d'ancrage immobiles.
Dans cette théorie, une augmentation de $C_{\mathrm{d}}$ n'entraîne pas de variation sur $l$, en effet, les longueurs libres de dislocation $l$, pour s'incurver sous l'effet d'une contrainte appliquée, doivent drainer leur nuage d'impuretés, ce qui entraîne l'existence d'une déformation retardée donc d'une déformation anélastique. Dans ce modèle, les impuretés piégées par la dislocation ne constituent pas des points d'ancrage immobiles. Ils ne doivent jouer aucun rôle sur $\Delta_{\text {S.K. }}$. mais seulement sur $\tau_{\text {s.K. }}$ [16].

Si l'on veut cependant expliquer la diminution de $\Delta_{\text {s.K. }}$, on peut admettre que certains des atomes d'oxygène appartenant au nuage d'interstitiels de la dislocation peuvent se regrouper pour constituer des ancrages efficaces.

L'introduction de $\tau_{2}, \tau_{3}, \ldots$ dans le spectre de relaxation de Snoek (Fig. 4) et les valeurs trouvées pour $\beta T_{\mathrm{c}}$ appuient cette possibilité.

Si pour simplifier, on suppose alors que $l$ varie linéairement avec $C_{\mathrm{d}}$ (dans un calcul élémentaire, on ne tiendra pas compte de la répartition statistique des valeurs de $l$ ) :

$$
l=b\left(1-\delta C_{\mathrm{d}}(t)\right),
$$

$\delta$ étant une constante, on pourra, avec (7), (14) et (15) exprimer $\Delta_{\mathbf{S} . K}$. en fonction de $\Delta_{\mathrm{s}}$. On trouve :

$$
\Delta_{\text {S.K. }}=\beta \Lambda b^{2}\left(1-\delta C_{\mathrm{T}}\right)^{2}\left(1+\frac{\delta \Delta_{\mathrm{S}}\left(T-\beta T_{\mathrm{c}}\right)}{\alpha\left(1-\delta C_{\mathrm{T}}\right)}\right)^{2},
$$

avec $\delta C_{\mathrm{T}} \leqq \leqq 1$, se réduit à :

$$
\Delta_{\text {S.K. }}=\beta \Lambda b^{2}\left[1+\frac{\delta \Delta_{\mathrm{s}}\left(T-\beta T_{\mathrm{c}}\right)}{\alpha}\right]^{2} .
$$

La vérification de ce modèle s'effectue en mettant la relation (18) sous la forme (19) :

$\Delta_{\mathrm{S} . \mathrm{K} .}^{1 / 2}=\left(\beta \Lambda b^{2}\right)^{1 / 2}+\frac{\left(\beta \Lambda b^{2}\right)^{1 / 2} \delta}{\alpha}\left(T-\beta T_{\mathrm{c}}\right) \Delta_{\mathrm{s}}$,

qui montre qu'il existe une relation linéaire entre $\Delta_{\mathbf{S} . K}^{1 / 2}$ et $\left(T-\beta T_{\mathrm{c}}\right) \Delta_{\mathrm{S}}$. Cette représentation est réalisée sur la figure $8 b$, pour quelques isothermes et avec $\beta T_{\mathrm{c}}=220 \mathrm{~K}$. L'ordonnée à l'origine permet d'obtenir une première évaluation de la densité de dislocations épinglées $\Lambda: \Lambda=\frac{\Delta_{\text {S.K. }}(0)}{\beta b^{2}}$. Avec $\beta=0,1$, on aboutit à $\Lambda \simeq 1,2 \times 10^{12}$ lignes. $\mathrm{cm}^{-2}$ pour $\varepsilon=45 \%$, valeur tout à fait acceptable et comparable à celle obtenue par Harper [25] dans Fe-C-N pour $\varepsilon=15 \%$, à savoir $\Lambda=3,7 \times 10^{11}$ lignes. $\mathrm{cm}^{-2}$. De plus, à l'aide de la pente de la représentation $8 b$ et de la valeur de $\alpha$ déduite de (7), on trouve $\delta \simeq 55$, ce qui permet de vérifier la condition $\delta C_{\mathrm{T}} \simeq 0,065 \leqq \leqq 1$.

Pour des temps de vieillissement suffisamment longs, dans le domaine de saturation de la loi (13), nous avons tracé dans la représentation d'Arrhénius 
de la figure 2, l'évolution du temps de relaxation du mécanisme de Snoek (droite (3)). Nous reportons également les points obtenus par la technique du retour de limite d'élasticité sur des échantillons identiques [19-20] et montrons ainsi que l'accord est satisfaisant. On trouve $\tau_{0}=1,3 \times 10^{-16} \mathrm{~s}$ pour $W_{\mathrm{S}}=1,166 \times 10^{5} \mathrm{~J} \cdot \mathrm{mole}^{-1}$. On constate alors, pour ce matériau de pureté commerciale, écroui puis vieilli, que l'énergie d'activation du mécanisme de Snoek est légèrement supérieure à celle d'un matériau pur non écroui tandis que $\tau_{0}$ est plus faible. En accord avec Schoeck et Seeger [29], nous pensons que cette observation montre que dans le champ de contrainte des dislocations, la remise en ordre du type Snoek subit quelques altérations notables, à savoir, une légère augmentation de l'énergie d'activation due à l'interaction élastique entre dislocation et atome de soluté et certainement une augmentation de la fréquence d'oscillation de ces atomes autour de leur position d'équilibre associée à une diminution de la distance à parcourir pour se réordonner.

Pour des temps de relaxation très longs et pour les plus hautes températures étudiées, il apparaît un nouveau maximum $H(\tau)$ qui peut être identifié au mécanisme de Snoek imputable aux atomes d'azote (droite (4), Fig. 2). Pour cette droite, on obtient $\tau_{0}=3,9 \times 10^{-15} \mathrm{~s}$ et $W_{\mathrm{s}}=1,502 \times 10^{5} \mathrm{~J} \cdot \mathrm{mole}^{-1}$, valeurs très proches de celles obtenues récemment par Boratto et Reed-Hill [30]. On peut noter, qu'à notre connaissance, ces mesures de temps de relaxation de l'azote à des températures aussi basses, sont uniques.

3.2 Relaxation dans le tantale. - Pour ne pas allonger inutilement le texte, nous présentons de façon plus synthétique les résultats obtenus sur le tantale. En général, on retrouve les différents phénomènes précédemment décrits pour le niobium.

i) Cas du tantale pur. - Quelques essais de relaxation et de frottement interne ont été réalisés sur des échantillons de tantale pur recuit (Tableau Ic). Les résultats concernant le réarrangement de Snoek de l'oxygène sont reportés sur la figure 9 (droite (1)) et sont justifiables d'une loi d'Arrhénius telle que

$W_{\mathrm{S}}=1,077 \times 10^{5} \mathrm{~J} . \mathrm{mole}^{-1}$ et $\tau_{0}=5,8 \times 10^{-15} \mathrm{~s}$.

Compte tenu de la dérive observée en torsion et imputable certainement au tréfilage lors de l'élaboration des fils, il ne nous a pas été possible d'étudier de manière précise l'évolution de l'amplitude relaxée avec la température.

ii) Cas du tantale commercial; influence de la concentration en oxygène. - Différents traitements de recuit de dopage sur du tantale de pureté commerciale ont été réalisés conformément au tableau $\mathrm{I} d$. On reporte sur la figure 10, l'évolution avec la teneur en oxygène des premières et secondes approximations des spectres de relaxation. On constate ainsi que la seconde appro-

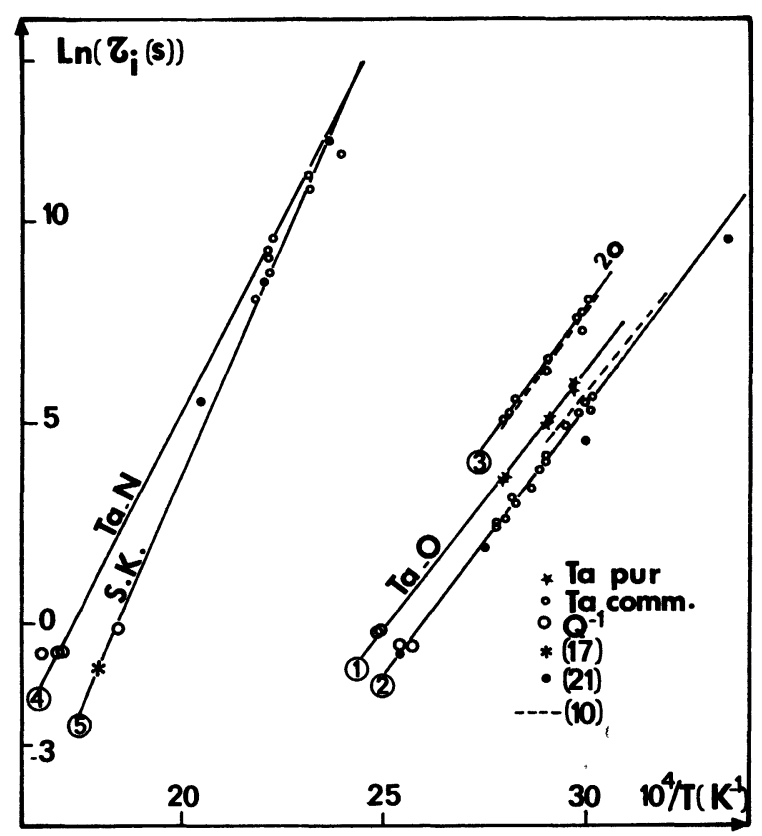

Fig. 9. - Diagramme d'Arrhénius correspondant aux temps de relaxation $\tau_{i}$ des différents phénomènes observés dans le tantale.

[Arrhenius diagram for the $\tau_{i}$ relaxation times of the various observed phenomena in $\mathrm{Ta}$.]

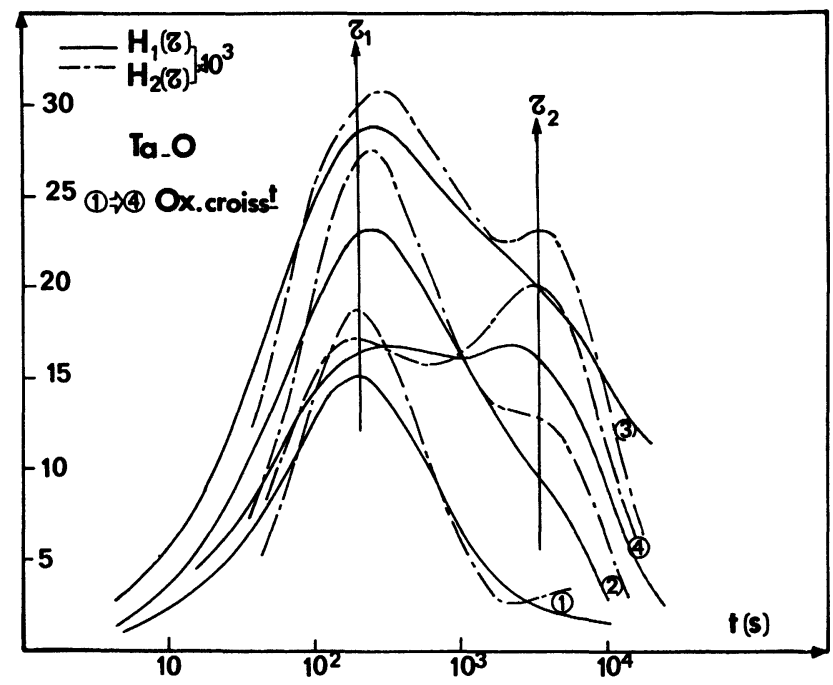

Fig. 10. - Evolution des deux premières approximations du spectre de relaxation avec la croissance de la teneur en oxygène. Positionnement des temps $\tau_{1}$ et $\tau_{2}$ des configurations 0 et 20 .

[Evolution of the two first approximations of the relaxation spectrum when the oxygen content increases. Positions of relaxation times of the 0 and 20 configurations.]

ximation $H_{2}(\tau)$ permet de séparer directement les relaxations imputables aux oxygènes simples et appariés $\left(\tau_{1}\right.$ et $\tau_{2}$, Fig. 10). Plusieurs tests ont été réalisés pour différentes isothermes et ont permis d'aboutir aux droites (2) et (3) de la figure 9, en très bon accord 
avec les résultats de Powers et Doyle [10] (droites en pointillé). Pour les oxygènes simples et appariés on obtient respectivement $W_{\mathrm{S}}=1,125 \times 10^{5}$ et $1,147 \times 10^{5} \mathrm{~J} \cdot \mathrm{mole}^{-1}$ pour $\tau_{0}=4,9 \times 10^{-16}$ et $2,4 \times 10^{-15} \mathrm{~s}^{1}$.

iii) Influence de lécrouissage. - Des fils de tantale commercial ont été recuits puis écrouis à $40 \%$ conformément au traitement du tableau $\mathrm{I} e$, puis ont subit plusieurs relaxations successives. On retrouve les différents phénomènes de vieillissement observés sur le niobium, à savoir, la décroissance de l'amplitude de la relaxation du mécanisme de Snoek associée au déplacement et la décroissance de l'amplitude du pic d'écrouissage. Ainsi, pour un temps de vieillissement très long, la droite d'Arrhénius du pic S.K. (droite (5), Fig. 9) est justifiable de $W_{\text {s.K. }}=1,864 \times 10^{5} \mathrm{~J} \cdot \mathrm{mole}^{-1}$ et $\tau_{0}=1,18 \times 10^{-18} \mathrm{~s}$, alors que celle du pic de Snoek tend à se confondre avec la droite (2) obtenue précédemment. On reporte également les points obtenus sur les mêmes échantillons par la technique du retour de limite d'élasticité [21], la cohérence étant assez bonne. Cependant, pour le pic S.K., on peut remarquer que les temps de relaxation mesurés sont très proches et se confondent quelque peu avec les temps de relaxation de Snoek de l'azote dans le tantale (droite (4), Fig. 9) dont l'énergie et le facteur de fréquence sont respectivement $W_{\mathrm{S}}=1,574 \times 10^{5} \mathrm{~J} \cdot \mathrm{mole}^{-1}$ et $\tau_{0}=5,3 \times 10^{-15} \mathrm{~s}$.

4. Application à la diffusion. - Si l'on admet que les atomes se réorientent dans les sites octaédriques les plus proches, il est possible de calculer les coefficients de diffusion de l'oxygène et de l'azote dans le niobium et le tantale. En effet, à l'aide d'un calcul élémentaire, on montre que

$$
D=\frac{a^{2}}{36 \tau}
$$

$a$ étant le paramètre du réseau cubique. Sur la figure 11, les résultats présentement obtenus à l'aide de (20) sont confrontés aux données récentes de Boratto et ReedHill [30]. On constate que l'accord est assez bon et confirme ainsi les valeurs calculées à basses températures. Cependant, comme nous venons de le voir, la teneur en oxygène et le taux d'écrouissage du matériau sont deux paramètres qui affectent aussi bien le facteur de fréquence que l'énergie d'activation de la relaxation de Snoek, ce qui peut expliquer la légère différence existant entre les deux études (Fig. 11). Quelques travaux très récents [31-32] tendent à extrapoler vers les hautes températures $\left(T \simeq 800^{\circ} \mathrm{C}\right)$ les résultats des mesures de traînage anélastique qui, confrontées aux résultats des expériences classiques de diffusion de masse à haute température (profil de pénétration), montrent que la loi d'Arrhénius n'est pas parfaitement respectée et s'incurve légèrement avec la température. L'énergie d'activation pour les hautes températures est supérieure de $0,12 \mathrm{eV}$ de celle obtenue à plus basses températures. L'écart ainsi obtenu est faible, de

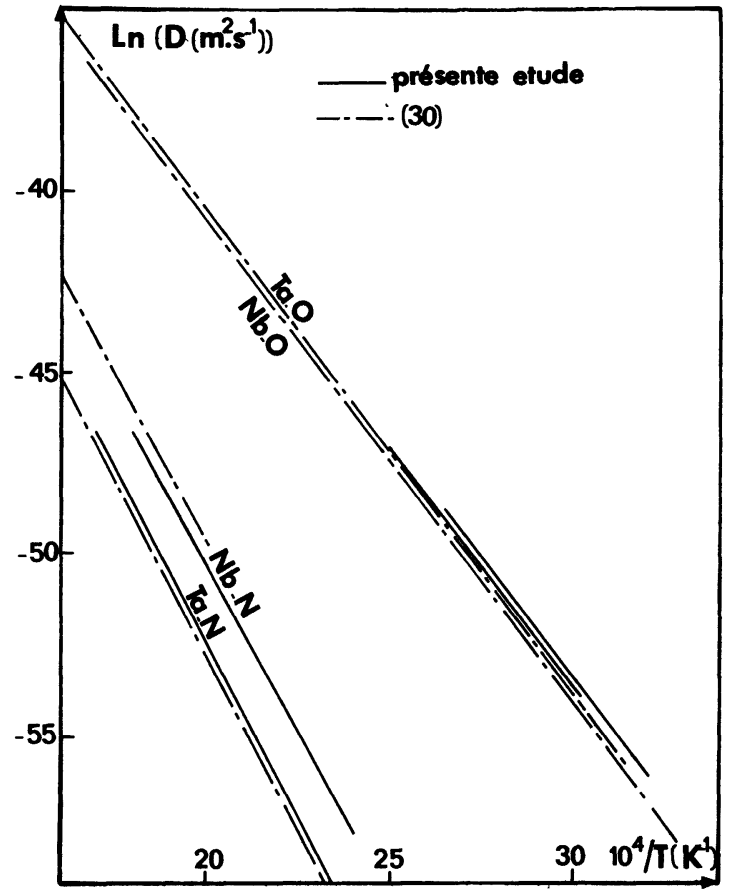

Fig. 11. - Evaluation des coefficients de diffusion de l'oxygène et de l'azote dans le niobium et le tantale. Confrontation avec la littérature.

[Estimation of the diffusion coefficients of oxygen and nitrogen in niobium and tantalum. Comparison with litterature results.]

l'ordre de 5 pour une dynamique de $10^{16}$ sur le coefficient de diffusion $D$, et sa confirmation nécessite une très grande précision et la stabilité du temps de relaxation, ce qui n'est pas forcément réalisé puisque $\tau_{\mathbf{r}}$ est extrêmement sensible au dopage et à l'écrouissage. Il faut remarquer que si l'on extrapole vers les hautes températures nos résultats trouvés sur le niobium commercial écroui, la droite ainsi calculée passe effectivement par le nuage de points reporté dans la littérature et correspondant à la diffusion de masse [3132]. Ainsi, en accord avec Boratto et Reed-Hill [33], nous pensons que la déviation de la loi d'Arrhénius vers les hautes températures n'est pas pleinement justifiée et nécessiterait une confirmation expérimentale sur des échantillons identiques.

5. Conclusions. - La présente étude, au voisinage de l'ambiante $\left(30 \leqq T \leqq 160^{\circ} \mathrm{C}\right)$, des mécanismes de relaxation dans le niobium et le tantale de diverses puretés et d'écrouissage variable peut se résumer à l'aide du tableau IV donné ci-après.

De plus, nous avons montré que pour le niobium pur recuit, l'amplitude totale relaxée décroissait avec la température en suivant une loi du type Curie-Weiss $\operatorname{avec} \beta T_{\mathrm{c}} \simeq 220 \mathrm{~K}$ pour $C \simeq 200 \mathrm{ppm}$ (équation (7)). Lorsque la teneur en oxygène croît, il a été mis en évidence l'apparition des relaxations des différentes configurations des oxygènes (20 et 30$)$. Ainsi, on a pu 
Tableau récapitulatif IV

\begin{tabular}{|c|c|c|c|c|c|c|c|c|}
\hline & \multicolumn{2}{|c|}{$\begin{array}{l}\text { Snoek oxygène } \\
\text { ox. }\end{array}$} & \multicolumn{2}{|c|}{2 ox. } & \multicolumn{2}{|c|}{ Snoek azote } & \multicolumn{2}{|c|}{$\begin{array}{l}\text { Snoek-Koster } \\
\text { pour } t \rightarrow \infty\end{array}$} \\
\hline & $\begin{array}{c}W_{s} \times 10^{5} \\
\left(\mathrm{~J} . \mathrm{mole}^{-1}\right)\end{array}$ & $\tau_{0}(\mathrm{~s})$ & $W_{\mathrm{s}} \times 10^{5}$ & $\tau_{0}$ & $W_{\mathrm{S}} \times 10^{5}$ & $\tau_{0}$ & $W_{\mathrm{s}} \times 10^{5}$ & $\tau_{0}$ \\
\hline $\begin{array}{l}\mathrm{Nb}-\mathrm{O} \text { pur, non } \\
\text { écroui }\end{array}$ & $1,04(5)$ & $1,9 \times 10^{-14} \mid$ & $T=86^{\circ} \mathrm{C}$ & $\mathrm{C}, \tau=200 \mathrm{~s}$ & - & - & 一 & - \\
\hline $\begin{array}{lr}\mathrm{Nb}-\mathrm{O}-\mathrm{N} & \text { com- } \\
\text { mercial } & \text { et } \\
\text { écroui } & \end{array}$ & $1,16(6)$ & $\left|1,3 \times 10^{-16}\right|$ & - & - & $1,50(2)$ & $3,9 \times 10^{-15}$ & $1,97(5)$ & $\times 10^{-22}$ \\
\hline $\begin{array}{l}\text { Ta-O pur, non } \\
\text { écroui }\end{array}$ & $1,07(7)$ & $5,8 \times 10^{-15}$ & 一 & - & - & 一 & - & - \\
\hline $\begin{array}{l}\text { Ta-O-N com- } \\
\text { mercial, plus } \\
\text { écroui }\end{array}$ & $1,12(5)$ & $4,9 \times 10^{-16}$ & $1,14(7)$ & $2,4 \times 10^{-15}$ & $1,57(4)$ & $5,32 \times 10^{-15}$ & $1,86(4)$ & $1,18 \times 10^{-18}$ \\
\hline
\end{tabular}

montrer que dès $450 \mathrm{ppm}$, l'influence de la relaxation des doublets d'oxygène sur le temps de relaxation des oxygènes libres n'est pas négligeable.

L'écrouissage, par l'intermédiaire de la diffusion des atomes d'oxygène vers les dislocations, joue un rôle important sur l'amplitude de la réorientation de Snoek; cet effet se traduisant par une décroissance simultanée et sensiblement linéaire de l'amplitude de Snoek et du
« pic » d'écrouissage (S.K.). Le facteur de fréquence et l'énergie du mécanisme de Snoek sont également sensibles au taux d'écrouissage (Tableau IV).

Les mesures effectuées à basses températures sont donc en bon accord avec les résultats disponibles dans la littérature, mais une attention particulière doit être apportée au taux de dopage et d'écrouissage lors de la mesure des temps de relaxation.

\section{Bibliographie}

[1] SNoeK, J. L., Physica 8 (1941) 711.

[2] Powers, R. W., Acta Metall. 3 (1955) 135-139.

[3] Powers, R. W. et Doyle, M. V., Trans. AIME J. Met. 211 (1957) 1285-1288.

[4] Szkopiak, Z. C. et Eliasz, W., J. Less-Common. Met. 11 (1966) 273-285.

[5] Ahmad, M. S. et Szkopiak, Z. C., J. Phys. Chem. Solids 31 (1970) 1799-1804.

[6] Ahmad, M. S. et Szkopiak, Z. C., J. Mat. Sci. 7 (1972) 701-707.

[7] Gibala, R. et Wert, C. A., Acta Metall. 14 (1966) 1095-1103-1113.

[8] Boratto, F. J. M. et ReEd-Hill, R. E., Scr. Metall. 11 (1977) 1107-1111.

[9] Boratto, F. J. M. et Reed-Hill, R. E., Scr. Metall. 11 (1977) 709-712.

[10] Powers, R. W. et Doyle, M. V., Trans. AIME 215 (1959) 655-665.

[11] Varchon, D., Oytana, C. et Delobelle, P., Phys. Status Solidi 47 (1978) 259-269.

[12] WoIrgard, J., Scr. Metall. 11 (1977) 641-643.

[13] Nowick, A. S. et Berry, B. S., Anelastic Relaxation in Crystalline solids (Academic Press, New York and London) 1972.

[14] Varchon, D., Oytana, C. et Lallement, G., Proc. VII Int. Cong. Rheology, Göteborg (1976) 434435.

[15] Lallement, G., C. R. Hebd. Séan. Acad. Sci. 268 (1969) 413-416.

[16] Oytana, C. et Varchon, D., Acta Metall. 27 (1979) 17-23.
[17] De Lamotte, E. et Wert, C., J. Phys. Soc. Japan 19, 9 (1964) $1560-1564$.

[18] Boone, D. H. et Wert, C., J. Phys. Soc. Japan 18 (1963) 141-146.

[19] Delobelle, P. et Oytana, C., Scr. Metall. 12 (1978) 765-770.

[20] Delobelle, P., Oytana, C. et Varchon, D., Mat. Sci. Eng. 29 (1977) 261-269.

[21] Delobelle, P., Oytana, C. et Varchon, D., Mém. Sci. Rev. Met. 75, 3 (1978) 175-185.

[22] Hartley, S., Acta Metall. 14 (1966) 1237-1246.

[23] Rosinger, H. E., Craig, G. B. et Bratina, W. J., Mat. Sci. Eng. 5 (1970) 163-169.

[24] Rosinger, H. E., Met. Sci. 9 (1975) 1-6.

[25] Harper, S., Phys. Rev. 63 (1951) 709-712.

[26] Rosinger, H. E., Craig, G. B. et Bratina, W. J., Philos. Mag. 25 (1972) 1331-1343.

[27] Friedel, J., Dislocations (Pergamon Press, Londres) 1964.

[28] SchoecK, G., Acta Metall. 11 (1963) 617.

[29] SchoecK, G. et Seeger, A., Acta Metall. 7 (1959) 469-477.

[30] Boratto, F. J. M. et Reed-Hill, R. E., Met. Trans. 8 (1977) 1233-1238.

[31] Farraro, R. et Mclellan, R. B., Mat. Sci. Eng. 39 (1979) 47-56.

[32] Mclellan, R. B., Mat. Sci. Eng. 45 (1980) 289-291.

[33] Boratto, F. J. M. et Reed-Hill, R. E., Mat. Sci. Eng. 43 (1980) 97-98. 